\title{
Pattern Recognition Control Design
}

Elisabeth Gambone

November 10, 2016

Spacecraft control algorithms must know the expected spacecraft response to any command to the available control effectors, such as reaction thrusters or torque devices. Spacecraft control system design approaches have traditionally relied on the estimated vehicle mass properties to determine the desired force and moment, as well as knowledge of the effector performance to efficiently control the spacecraft. A pattern recognition approach can be used to investigate the relationship between the control effector commands and the spacecraft responses. Instead of supplying the approximated vehicle properties and the effector performance characteristics, a database of information relating the effector commands and the desired vehicle response can be used for closed-loop control. A Monte Carlo simulation data set of the spacecraft dynamic response to effector commands can be analyzed to establish the influence a command has on the behavior of the spacecraft. A tool developed at NASA Johnson Space Center (Ref. 1) to analyze flight dynamics Monte Carlo data sets through pattern recognition methods can be used to perform this analysis. Once a comprehensive data set relating spacecraft responses with commands is established, it can be used in place of traditional control laws and gains set. This pattern recognition approach can be compared with traditional control algorithms to determine the potential benefits and uses.

1. Carolina I. Restrepo and John E. Hurtado. "Tool for Rapid Analysis of Monte Carlo Simulations", Journal of Spacecraft and Rockets, Vol. 51, No. 5 (2014), pp. 1564-1575. 